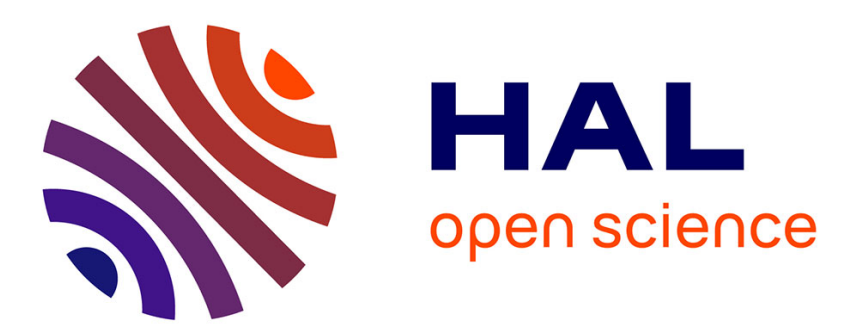

\title{
Réflecto-interféromètre simplifié à branche ouverte pour la mesure des permittivités complexes dans la bande des $8 \mathrm{~mm}$
}

\author{
A. Bottreau, Cl. Marzat
}

\section{- To cite this version:}

A. Bottreau, Cl. Marzat. Réflecto-interféromètre simplifié à branche ouverte pour la mesure des permittivités complexes dans la bande des $8 \mathrm{~mm}$. Revue de Physique Appliquée, 1971, 6 (2), pp.215216. 10.1051/rphysap:0197100602021500 . jpa-00243529

\section{HAL Id: jpa-00243529 https://hal.science/jpa-00243529}

Submitted on 1 Jan 1971

HAL is a multi-disciplinary open access archive for the deposit and dissemination of scientific research documents, whether they are published or not. The documents may come from teaching and research institutions in France or abroad, or from public or private research centers.
L'archive ouverte pluridisciplinaire HAL, est destinée au dépôt et à la diffusion de documents scientifiques de niveau recherche, publiés ou non, émanant des établissements d'enseignement et de recherche français ou étrangers, des laboratoires publics ou privés. 


\title{
RÉFLECTO-INTERFÉROMÈTRE SIMPLIFIÉ A BRANCHE OUVERTE POUR LA MESURE DES PERMITTIVITÉS COMPLEXES DANS LA BANDE DES $8 \mathrm{~mm}$
}

\author{
par A. BOTTREAU et Cl. MARZAT
}

Laboratoire d'Optique Ultra-Hertzienne, Faculté des Sciences de Bordeaux

\begin{abstract}
Résumé. - Les auteurs décrivent un interféromètre à branche ouverte permettant de mesurer le coefficient de réflexion complexe d'un diélectrique placé sur un miroir. Des tables numériques indépendantes de la longueur d'onde, qui tiennent compte des réflexions multiples internes aux échantillons, donnent la permittivité complexe $\left(\varepsilon=\varepsilon^{\prime}-j \varepsilon^{\prime \prime}\right)$ à partir des valeurs mesurées.
\end{abstract}

\begin{abstract}
The authors describe an open branch interferometer which can measure the complex reflection coefficient of a dielectric set on a mirror. Numerical tables independant of the wave length and taking into account multiple internal reflections of samples give the complex permittivity $\left(\varepsilon=\varepsilon^{\prime}-j \varepsilon^{\prime \prime}\right)$ from measured values.
\end{abstract}

I. Description du réflecto-interféromètre. — L'onde $\left(\lambda_{0}=8,9 \mathrm{~mm}\right)$ émise par un klystron stabilisé en fréquence [3], [5], se réfléchit en partie sur l'iris de couplage de l'aérien pour constituer l'onde de référence ; tandis que la partie de l'onde transmise effectue une double traversée de l'échantillon avec une réflexion sur le miroir porte-échantillon: c'est l'« onde de mesure ".

Le mélange des deux ondes se fera dans le coupleur directif $3 \mathrm{~dB}(5$, Fig. 1$)$ et en faisant varier la distance à l'air libre (aérien-miroir), on fera défiler les franges interférométriques, que l'on détectera avec le cristal monté directement sur le coupleur directif.

On trouvera un schéma du réflecto-interféromètre sur la figure 1.

Pour réduire les dimensions de l'échantillon, il faut prévoir une optique focalisante. Afin de limiter au maximum les réflexions multiples indésirables au niveau de l'aérien, celui-ci est constitué par un cornet cylindrique entièrement rempli de lucoflex $\left(\varepsilon^{\prime}=2,76\right.$, $\left.\varepsilon^{\prime \prime}=0,024\right)$ formant lentille de concentration d'énergie. Ce dispositif d'une longueur de $115 \mathrm{~mm}$ assure par ailleurs un découplage de $5 \mathrm{~dB}$ tout en donnant un taux d'onde stationnaire $\theta=1,01$ du côté émission. L'étude de ce dispositif pourra être trouvée par ailleurs [1].

II. Méthode de mesure. - Il s'agit d'une méthode par substitution. En l'absence d'échantillon l'aérien a été étudié expérimentalement de manière à donner des franges interférométriques à minimums nuls au niveau du pseudo-foyer. Lorsque l'on place la substance à étudier sur le miroir, il y a un glissement des franges interférométriques; on déplace alors le miroir pour rattraper la frange initiale [2], [3] (Fig. 2).
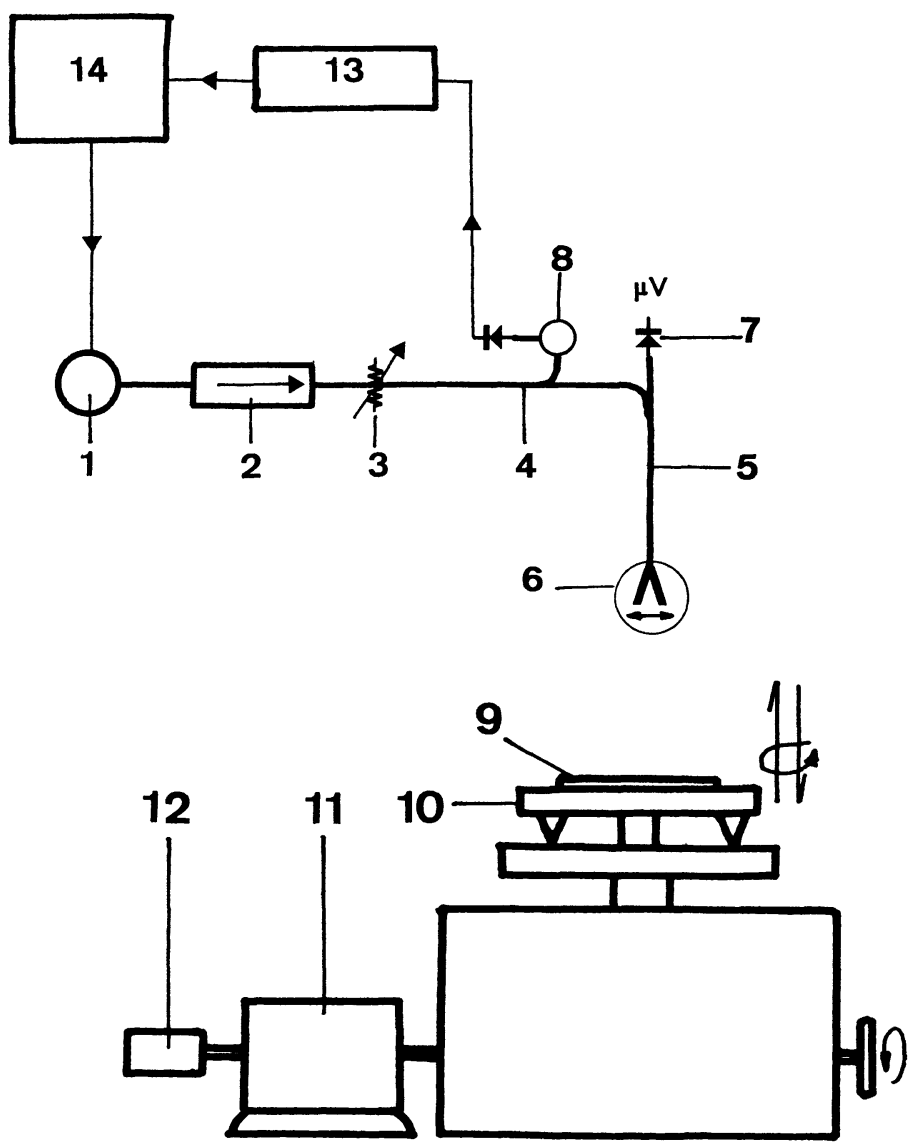

FIG. 1. - 1. Klystron. - 2. Isolateur à ferrite. - 3. Atténuateur. - 4. Coupleur directif $20 \mathrm{~dB}$. - 5. Coupleur directif $3 \mathrm{~dB}$. -6. Aérien. - 7. Cristal détecteur. - 8. Cavité de stabilisation. - 9. Echantillon. - 10. Miroir porte-échantillon. - 11 . Moteur. - 12. Compte-tour. - 13. Stabilisation de fréquence. - 14. Alimentation klystron. 


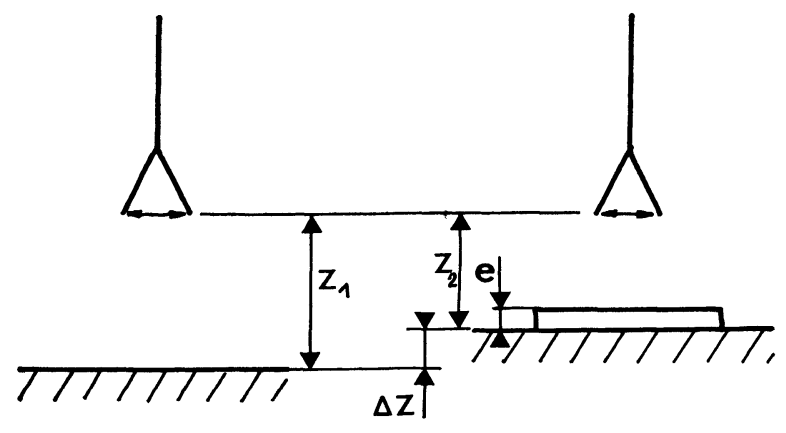

FIG. 2.

De la mesure de ce déplacement et du taux d'onde stationnaire on en déduit alors le coefficient de réflexion complexe [2], [3] $R=|R| \exp (-j \psi)$ qui compte tenu des réflexions multiples internes à l'échantillon plans parallèles d'épaisseur $e$ s'écrit :

$$
R=\frac{\rho-\exp (-2 \gamma e)}{1-\rho \exp (-2 \gamma e)}
$$

avec

$$
\rho=\frac{\beta_{0}-\beta+j \alpha}{\beta_{0}+\beta-j \alpha}
$$

et $\gamma=\alpha+j \beta$ (constante de propagation).

Pour déterminer la permittivité complexe

$$
\varepsilon=\frac{\beta^{2}-\alpha^{2}}{\beta_{0}^{2}}-J \frac{2 \alpha \beta}{\beta_{0}^{2}}
$$

nous avons réalisé des tables [4] indépendantes de la longueur d'onde et du mode de propagation (libre ou guidée). A partir des résultats expérimentaux on a alors directement les valeurs de $\varepsilon^{\prime}$ et de $\varepsilon^{\prime \prime}$.

III. Mesures de recoupement. - Afin de tester l'appareil, nous avons vérifié sur des panneaux industriels (téflon, polystyrène expansé, altuglas, lucoflex, bakélite, dellite, canevasite) que les résultats obtenus étaient en accord avec ceux donnés par d'autres méthodes interférométriques [2], [3]. La précision obtenue est de 2 à $5 \%$ sur $\varepsilon^{\prime}$ et de 3 à $8 \%$ sur $\varepsilon^{\prime \prime}$ suivant l'épaisseur de l'échantillon.
Nous avons également fait une étude systématique du déphasage en fonction de l'épaisseur pour différents matériaux (Fig. 3). Les courbes obtenues ondu-

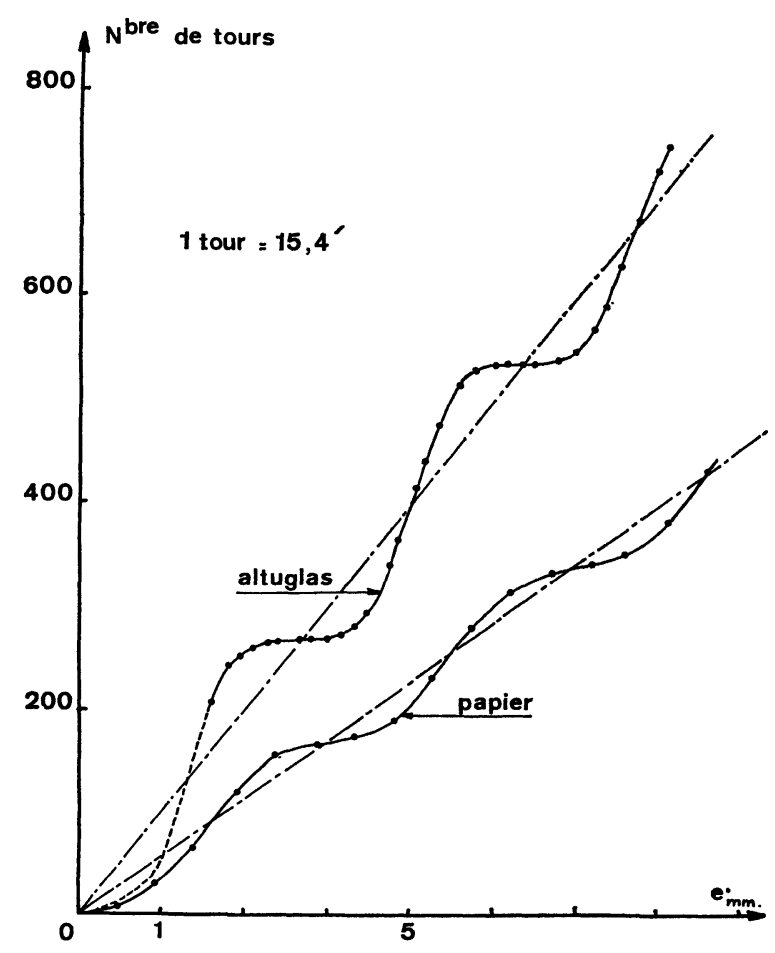

FIG. 3.

lent autour des droites correspondantes à l'absence de réflexions multiples conformément à la théorie [2], [3] et aux valeurs données par les tables [4]. Pour les milieux fortement absorbants, un abaque permet de déterminer $\varepsilon^{\prime}$ et $\varepsilon^{\prime \prime}$ à partir des résultats expérimentaux.

IV. Conclusion. - Nous avons mis au point un réflecto-interféromètre à branche ouverte de conception très simple, qui permet, sans aucun réglage délicat de déterminer la permittivité complexe d'un matériau plans parallèles sans usinage préalable de celui-ci. Il peut être adapté sans difficulté aux mesures sur les liquides, voire même sur les gaz ionisés.

\section{Bibliographie}

[1] Bottreau (A.), Marzat (Cl.), C. R. Acad. Sci., Paris 1970, 271 série $B, 233$.

[2] Botrreau (A.), Thèse de Doctorat ès Sciences, Bordeaux, 1969.

[3] Marzat (Cl.), Thèse de Doctorat ès Sciences, Bordeaux 1969.
[4] Bottreau (A.), Marzat (Cl.), Tables permettant de déterminer la permittivité d'un diélectrique par la méthode du miroir accolé, O. U. H., 1969.

[5] Servant (Y.), Thèse Doctorat ès Sciences, Bordeaux, 1968. 